\section{Meteorite impacts on humans and on buildings}

SIR-From nine years of observation with the Meteorite Observation and Recovery Project, a network of 60 cameras in western Canada, we have derived the frequency of metcorite falls on the Earth as a function of the total mass of meteorites to reach the ground for each event'. Assuming that the total mass of an event is twice the mass of the largest fragment observed by the cameras, we conclude that

$\log N=-0.689 \log m+2.967$

where $N$ is the number of events per year in which a mass of at least $m$ grams of meteorite is deposited in an area of $10^{\circ}$ $\mathrm{km}^{2}$. This formula predicts 39 events per year with a mass of at least $100 \mathrm{~g}$ in each million $\mathrm{km}^{2}$, or $5,8(0)$ such events on the total land area of the Earth.

Our present concern is to estimate the probability of persons or buildings being struck by meteorites. Our major assumptions are: (1) A human being occupies an area of $0.2 \mathrm{~m}^{2}$. (2) The smallest impact likely to be reported would involve a fragment of a few grams. (3) Typical meteorite falls consist of five major fragments. (4) Meteorite fragments larger than $200 \mathrm{~g}$ will normally penetrate a roof and ceiling. (We also assume that, if the total mass exceeds $500 \mathrm{~g}$, each of the five fragments could penetrate a roof, whereas none of the pieces from lesser events will do so.) (5) Residents of North America spend 5 per cent of each day outside and 95 per cent of the day protected by a roof. (6) The total roof area of buildings averages $50 \mathrm{~m}^{2}$ per member of population.

On the basis of these plausible assumptions we derive the frequency of impacts on people and on buildings and we wish to compare the values with recent experience. For this purpose we consider the population of the United States plus Canada (about $2.5 \times 10^{\circ}$ persons) since we expect news coverage of relatively minor events to be more complete for this sample than in many densely populated areas of the world. For this North American sample. our assumptions predict an annual rate of 0.0055 impacts on people (one event per 180 years) and 0.80 impacts per year causing damage to buildings.

The only documented case of a person being struck by a meteorite appears to be a fall on 30 November 1954:. A fragment of a stony meteorite weighing $3.9 \mathrm{~kg}$ penetrated the roof and ceiling of a house in Sylacauga. Alabama, bounced off a large radio and struck a women who was asleep on a couch, inflicting painful bruises. At first glance it would appear unlikely that there would be even one known event only 31 years ago, but the fact that there are no other verified cases elsewhere in the world indicates that impacts on people are extremely rare.

The prediction for impacts on buildings is more readily verified because the pre- dicted rate is much higher. All known meteorite falls and chance recoveries are reported in the Meteoritical Bulletin, published about once per year in the journal Meteoritics. During the past 20) years, there are reports of 16 recoveries from fresh meteorite falls in the United States and Canada. For seven of these events, there was appreciable damage to some building, usually the roof of a home or garage. In the Louisville, Kentucky fall in January 1977, three separate buildings were struck, so there are nine reports of damage. Two other events, which we have not included, involved a small meteorite which caused no damage to a roof and a $1.3 \mathrm{~kg}$ object that damaged a mailbox. It is clear that impact on a building greatly increases the probability of recovery of the meteorite, since only a very small fraction of all falls will strike buildings, whereas half of the recent recoveries have such an involvement.

We would predict 16 damaged buildings in 20) years for this sample, which is close to the nine reports of damage. Since we would not expect small meteorites to be located, identified and reported in all cases, especially for minor damage to commercial buildings where there might be little interest in finding the cause of a leaky roof, we suppose the actual rate may be somewhat higher than our estimate. Our guess of five effective fragments per event may be too low.

To extrapolate to the entire world population, estimated at 5,000 million, then the number of events will increase by a factor of 20 over the North American sample if we use the same assumptions. One would then expect a person to be struck by a meteorite once in nine years and that sixteen buildings per year would receive some damage from metcorites.

We thank Dr Paul A. Feldman of the Herzberg Institute for valuable discussions.

I. Hall.iday

A. T. BI.ACKWEI.L A. A. Griffin

Herzberg Institute of Astrophysics,

National Research Council of Canada. Ottawa, Canada KIA OR6

1. Halliday. I.. Blackweil. A.T. Griffin. A.A. Scieme 233, $1+115-1+107(1984)$.

Swindel. (i.W. Ji \& Jones. W. B. Mateoritics. 1. 125-1,32 (1054)

\section{A question of cellular immortality}

SiR-In "Human T-lymphotropic retroviruses" (Nature 316, 395-403; 1985), as well as other articles many authors now speak of the "immortalization" of cells by the expression of oncogenes. This term is generally used to refer to the ability of a cell line to grow in vitro indefinitely. without senescence or a growth crisis. The use of the term, to those not versed in the minutiae of oncogene nomenclature, would seem to imply specific gene(s) whose func- tion is to prevent cell senescence. This gene would seem to have no corresponding physiological function in vivo, however.

A more plausible explanation would be that the cell, at a certain point in its stepwise differentiation, fails to turn off a regulatory gene. The cell continues to express this regulatory gene and the genes whose expression it controls. The cell is "stuck" in a state of expressing inappropriate genes and is prevented from terminal differentiation. This observation would fit with the fact that established cell lines. while not necessarily tumorigenic, require fewer steps to render them tumorigenic, perhaps as little as a growth factor with a specific effect on a certain differentiation state.

Margaret Pillow Sillina Bendix

Bendix Environmental Research Inc.

Fox Plaza, Suite 902

1390 Market Street,

San Francisco, California 94102, USA

\section{Weak violation - a new concept in relativity?}

SIR-Quoting from Nature News and Views. "If there are violations of special relativity, they are at most weak violations, departures from what is not strict orthodoxy and not flat contradictions of it". Not everyone would agree with this statement. For many decades now. Einstein's relativity, and the need for absolute conformity with its basic tenets, have stood firmly in the way of any theoretical advance which dares to imply the slightest violation. But though it is improbable that we will ever see support for Marinov's claim, that the Earth's cosmic motion through space can be detected by tests confined to the laboratory. should this be demonstrated as proven, then Einstein's theory is violated by a death blow struck into its very heart.

Consistent with empirical data, a number of alternative laws of electrodynamics were shown by Clerk Maxwell to be derivable from a formula based on the relative velocity of interacting charges, taken in conjunction with what we would. today. term a "quantum electrodynamic" proposition (Fechner's hypothesis). Einstein"s constraint, that any such law must satisfy the test of covariance, excludes a form of law which has recently been supported by experimental evidence from anomalous forces accompanying electric discharges in electrolytic solutions ${ }^{-\ldots:}$

Consequently, research into practical applications of the indicated law has, at least until the present time, been retarded. blocked by the "inviolate" principle of relativity, and for the very reason that a successful outcome would be a strong violation and therefore improbable.

Whereas Marinov has sought to chal- 